\title{
Cyclopenta[1]phenanthrene titanium trichloride derivatives: syntheses, crystal structure and properties as catalysts for styrene polymerization
}

\author{
Nicole Schneider, Marc-Heinrich Prosenc, Hans-Herbert Brintzinger * \\ Fakultät für Chemie, Unicersität Konstanz, Unicersitätsstr. 10. Postfach 5560 M 737, D.78464 Konstanz, Germany
}

\begin{abstract}
Cyclopentall]phenanthrene titanium trichloride and its 2-methyl and phenyl derivatives were synthesized; the crystal structure of the 2-methyl-substituted complex was determined by $\mathrm{X}$-ray diffraction analysis. In the presence of methylalurnoxane (MAO), these complexes give highly active catalysts for the syndiotactic polymerization of styrene; the 2-phenyl-substituted complex exceeds all previously described catalysts in its catalytic activity.
\end{abstract}

\section{Introduction}

Since Ishihara and coworkers [1] discovered the syndiotactic polymerization of styrene with MAO-activated cyclopentadienyl titanium catalysts, the mechanism of the insertion reaction has been discussed controversially [2]. It seems to be established now that at temperatures above $0^{\circ} \mathrm{C}$ syndiotactic polystyrene (s-PS) is formed via a coordination-type Ziegler-Natta mechanism with a 15-electron Ti(III)-species being the active center [3]. This raises the question whether catalyst systems of this kind can be influenced in their properties by different cyclopentadienyl ligands in a manner similar to that observed in recent years for metallocene-based propene polymerization catalysts [4]. Recently, it has been shown that cyclopentadienyl systems with annelated six-rings

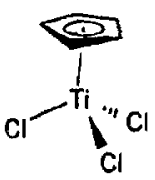

1

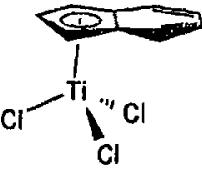

2

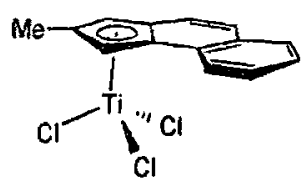

3
Fig. 1. Cyclopentadienyl titanium trichloride derivatives reperted by Ishihara and coworkers (complex 1) [1] and Rausch and coworkers (complexes 2 and 3) [5,6] to catalyze the syndiospecific polymerization of styrene in the presence of MAO.
(Fig. 1) are very active catalysts, which keep their productivity and syndiotacticity even at elevated temperatures $[5,6]$. In this paper we want to extend this correlation to three new catalysts 4,5 and 6 , which are derived from the cyclopenta[1]phenanthrene ligand system.

\section{Results and discussion}

Complexes 4, 5 and 6 were synthesized from their precursors 7,8 and 9 [7] following procedures reported for related compounds (Fig. 2).

The structure of complex $\mathbf{5}$, determined by an X-ray diffraction study (Fig. 3; Table 1), supports the closeto-tetrahedral geometry at the titanium center and the $\eta^{5}$-coordination of the annelated cyclopentadienyl ring.

Polymerizations were conducted by reacting styrene with complexes 4, 5 and 6 in the presence of MAO at
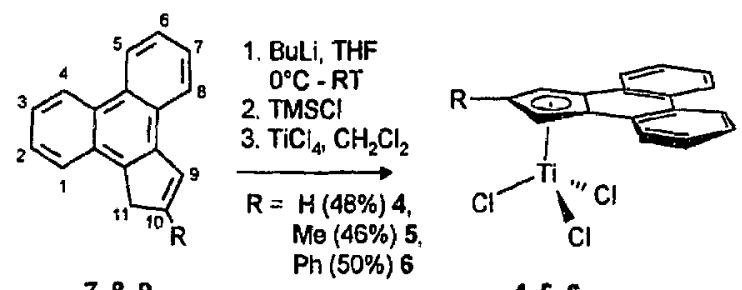

$4,5,6$

\footnotetext{
Corresponding author, Fax: +497531883137.
}

Fig. 2. Syntheses of complexes 4-6. 


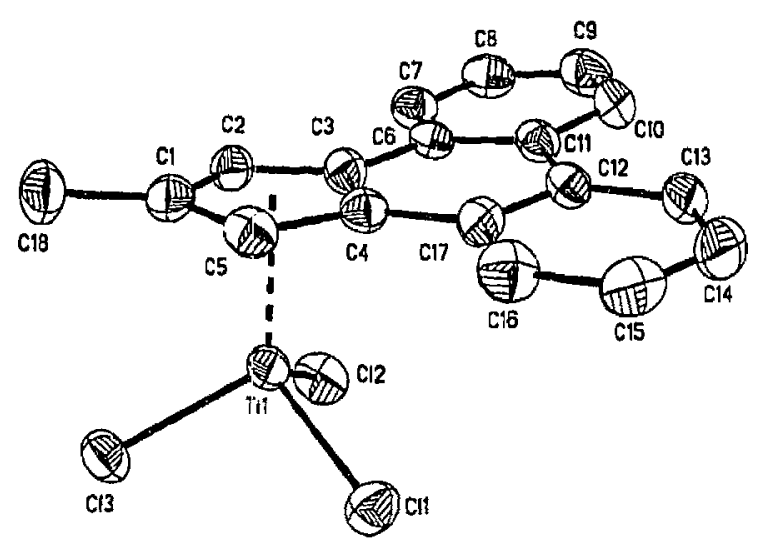

Fig. 3. Molecular structure of complex $\mathbf{5}$ ( $50 \%$ probability thermal elipsoids).

different temperatures in toluene. For comparison, complex 3 (synthesized according to [6]) was also used as a polymerization catalyst under identical conditions '. All complexes except 4 exhibit their highest activities around $75^{\circ} \mathrm{C}$. Above this temperature, deactivation seems to take place more readily [8]. The results (Table 2) show that complex 6 exhibits particularly high activities in the production of polystyrenes with high stereoregularity and molecular weight. This observation is probably due to the huge flat 'roof' over the active titanium tripod, which makes the titanium center sterically rather inaccessible; this steric congestion at the titanium center could possibly destabilize the formation of detrimental $\beta$-agostic interactions, which are thought to impede olefin insertions relative to chain termination by $\beta$-H-transfer. In addition, ' $\pi$-stacking' [9] between the aromatic ligand system and a coordinated styrene

\footnotetext{
'For complex 3 we find activities which are considerably higher than those reported by Foster et al. under the same conditions [6]. This observation might have its reason in different grades of MAO used by them and by us.
}

Table 1

Selected bond lengths $(\AA)$ and bond angles, $\left(^{\circ}\right)$ for complex 5 with estimated standard devistions

\begin{tabular}{|c|c|c|c|}
\hline \multicolumn{2}{|l|}{ Bond distances } & \multicolumn{2}{|l|}{ Bond angles } \\
\hline $\mathrm{T} ;(1)-\mathrm{Cl}(1)$ & $2.244(2)$ & $\mathrm{Cl}(1)-\mathrm{Ti}(1)-\mathrm{Cl}(2)$ & $104.70(6)$ \\
\hline $\mathrm{Ti}(1)-\mathrm{Cl}(2)$ & $2.235(2)$ & $\mathrm{Cl}(1)-\mathrm{Ti}(1)-\mathrm{Cl}(3)$ & $102.01(5)$ \\
\hline $\mathrm{Ti}(1)-\mathrm{Cl}(3)$ & $2.216(1)$ & $\mathrm{Cl}(2)-\mathrm{Ti}(1)-\mathrm{Cl}(3)$ & $102.75(6)$ \\
\hline Ti(i)-Cent:oid & 2.041 & $C(18)-C(1)-C e n t r o i d$ & 175.5 \\
\hline $\operatorname{Ti}(1)-C(1)$ & $2.374(4)$ & $\mathrm{Cl}(1)-\mathrm{Ti}(1)$-Centroid & 114.9 \\
\hline $\operatorname{Ti}(1)-C(2)$ & $2.329(4)$ & $\mathrm{Cl}(2)-\mathrm{Ti}(1)$-Cenntoid & 114.8 \\
\hline $\mathrm{Ti}(1)-\mathrm{C}(3)$ & $2.400(4)$ & $\mathrm{Cl}(3)-\mathrm{Ti}(1)$-Centroid & 116.0 \\
\hline $\mathrm{Ti}(1)-\mathrm{C}(4)$ & $2.409(4)$ & & \\
\hline $\operatorname{Ti}(1)-\mathrm{C}(5)$ & $2.344(4)$ & & \\
\hline
\end{tabular}

monomer might contribute to the high activity and stereospecificity.

\section{Experimental section}

All work involving air- or moisture-sensitive compounds was carried out using standard high-vacuum Schlenk or drybox techniques. Mass spectra were obtained using a Finnigan MAT 312 spectrometer. NMR spectra were recorded on Bruker AC $250 \mathrm{MHz}$, WM $250 \mathrm{MHz}$ or DRX $600 \mathrm{MHz}$ spectrometers (Internal standard $\left.\mathrm{CDCl}_{3}: 7.24 \mathrm{ppm} ; \mathrm{C}_{6} \mathrm{D}_{6}: 7.16 \mathrm{ppm}\right)$. The numbers in the NMR-data section refer to the labeling in Fig. 2.

Toluene and ether were disilled over $\mathrm{Na}$, pentane over $\mathrm{Na}-\mathrm{K}$-alloy and $\mathrm{CH}_{2} \mathrm{Cl}_{2}$ over $\mathrm{CaH}_{2}$ prior to use. Trimethylsilylchloride (Aldrich) was distilled over $\mathrm{CaH}_{2}$. MAO was donated by Witco GmbH. 2,3-Dihydro-2-oxo-1H-cyclopenta[1]phenanthrene [10] was synthesized according to literature procedures. By reaction of the latter in a Shapiro-reaction or by addition of Methyl-Grignard reagent and subsequent dehydrogenation, $1 \mathrm{H}$-cyclopenta[l]phenanthrene and $1 \mathrm{H}-2$-methylcyclopenta[1]phenanthrene were obtained in $47 \%$ and $85 \%$ yield [7].

Table 2

Polymerization of styrene with complexes 3-6 in the presence of MAO

\begin{tabular}{|c|c|c|c|c|c|c|c|c|c|c|c|c|}
\hline \multirow[t]{2}{*}{ Catalysator } & \multicolumn{4}{|c|}{$50^{\circ} \mathrm{C}$} & \multicolumn{4}{|c|}{$75^{\circ} \mathrm{C}$} & \multicolumn{4}{|c|}{$100^{\circ} \mathrm{C}$} \\
\hline & $A$ & SY & $M_{\mathrm{r}}$ & $T_{\mathrm{m}}$ & $A$ & SY & $M_{\mathrm{r}}$ & $T_{\mathrm{m}}$ & $\bar{A}$ & SY & $M_{\mathrm{r}}$ & $T_{\mathrm{m}}$ \\
\hline$R=H(4)$ & 4 & 64 & 6.9 & 246.4 & 7 & 49 & 4.2 & 241.0 & 41 & 88 & 1.9 & n.d. \\
\hline $\mathrm{R}=\mathrm{Me}(5)$ & 10 & 83 & 12.3 & 270.2 & 26 & 89 & 7.4 & 267.5 & 24 & 82 & 2.9 & 259.6 \\
\hline $\mathrm{R}=\mathrm{Ph}(6)$ & 40 & 85 & 27.7 & 267.8 & 75 & 92 & 13.0 & 265.2 & 45 & 90 & 5.8 & 264.9 \\
\hline \multirow[t]{2}{*}{ (2-Me-benz $[e]$ indene $) \mathrm{TiCl}_{3}(3)^{4}$} & 15 & 88 & 14.8 & 270.3 & 76 & 89 & 9.3 & 267.8 & 42 & 92 & 7.1 & 267.6 \\
\hline & $(18$ & 93 & 42.4 & 275.2) & $(15$ & 91 & 18.8 & $261.0)$ & $(7$ & 90 & 10.6 & 258.4) \\
\hline
\end{tabular}

$[\mathrm{Ti}]=50 \mu \mathrm{mol} / \mathrm{l}, \mathrm{Al}: \mathrm{Ti}=4000 \mathrm{l}$, [styrene] $=0.88 \mathrm{M}$, reaction time: $10-20 \mathrm{~min}$, activities $A\left[\times 10^{7} \mathrm{~g} \mathrm{PS} /(\mathrm{mol} \mathrm{Ti} \times \mathrm{mol} \mathrm{styrene} \times \mathrm{h})\right.$ ], syndiotacticity SY (\% insoluble in 2-butanone), molecular weight $M_{\mathrm{r}}\left(\times 10^{4}\right)$ determined by GPC, $T_{\mathrm{n}}\left({ }^{\circ} \mathrm{C}\right)$ determined by DSC.

${ }^{2}$ Data in parentheses from ref. [6] under identical conditions; molecular weights determined by intrinsic viscosity. 
Dif ferential scanning calorimetry (DSC) for melting transition $\left(T_{n 1}\right)$ and gel permeation chromatography (GPC) for molecular weight determinations were recorded at BASF laboratories.

\section{I. IH-2-Phenyl-cyclopentall]phenanthrene (9)}

$2 \mathrm{ml}(6 \mathrm{mmol})$ of a $3 \mathrm{M}$ solution of phenylmagnesiumbromide in ether (Aldrich) were added dropwise at $0^{\circ} \mathrm{C}$ to $1.0 \mathrm{~g}$ 2,3-dihydro-2-oxo- $1 \mathrm{H}$-cyclopenta[1]phenanthrene $(4.31 \mathrm{mmol})$ in $100 \mathrm{ml}$ toluene. After warming to room temperature, the reaction mixture was stirred for $2 \mathrm{~h}$ and then hydrolyzed with $50 \mathrm{ml}$ of a saturated aqueous solution of $\mathrm{NH}_{4} \mathrm{Cl}$ and extracted with $300 \mathrm{ml}$ of ether. The organic layers were extracted again with $100 \mathrm{ml}$ of brine, dried over $\mathrm{MgSO}_{4}$ and evaporated to dryness. The solid thus obtained was dissolved in $100 \mathrm{ml}$ of toluene and stirred with $100 \mathrm{mg}$ of $p$-toluene sulfonic acid under reflux for $2 \mathrm{~h}$. After addition of $50 \mathrm{ml}$ of a saturated aqueous solution of $\mathrm{NaHCO}_{3}$ and extraction with $200 \mathrm{ml}$ of ether, the organic layers were dried over $\mathrm{MgSO}_{4}$ and evaporated to dryness. Flash-chromatography (pentane/ethyl acetate $=50: 1$ ) and subsequent evaporation in vacuo gave 2-phenyl-cyclopenta[1]phenanthrene as colorless needles. 'H-NMR ( $\left.\mathrm{CDCl}_{3} ; 600 \mathrm{MHz}\right): \delta 8.02(\mathrm{~d}, J=7.7$ $\left.\mathrm{Hz}, \mathrm{C}_{1}-\mathrm{H}\right), 7.62-7.56\left(\mathrm{~m}, \mathrm{C}_{2.3}-\mathrm{H}\right), 8.68(\mathrm{~d}, J=8.2 \mathrm{~Hz}$, $\left.\mathrm{C}_{4}-\mathrm{H}\right), 8.72\left(\mathrm{~d}, J=7.1 \mathrm{~Hz}, \mathrm{C}_{5}-\mathrm{H}\right), 7.66-7.64(\mathrm{~m}$, $\left.\mathrm{C}_{6.7}-\mathrm{H}\right), 8.20\left(\mathrm{~d}, J=7.0 \mathrm{~Hz}, \mathrm{C}_{8}-\mathrm{H}\right), 7.79\left(\mathrm{~s}, \mathrm{C}_{9}-\mathrm{H}\right)$, $4.20\left(\mathrm{~s}, 2 \mathrm{C}_{11}-\mathrm{H}\right), 7.75(\mathrm{~d}, J=7.3 \mathrm{~Hz}, 2$ orthoPh-H), $7.42(\mathrm{t}, J=7.3 \mathrm{~Hz}, 2$ metaPh-H), $7.29(\mathrm{t}, J=7.3 \mathrm{~Hz}$, paraPh-H); assignments of the proton signals by DQFCOSY, ${ }^{13} \mathrm{C}$, HMQC and ROESY measurements, MS (EI): $m / z .292\left(\mathrm{M}^{+}, 100 \%\right), 215\left(\mathrm{M}^{+}-\mathrm{Ph}, 6 \%\right)$, Anal. Calcd for $\mathrm{C}_{23} \mathrm{H}_{16}: \mathrm{C}, 94.48$; $\mathrm{H}$, 5.52. Found: C, 94.66; H. 5.63 .

\subsection{General method for the synthesis of 1-trinethyl- silyl-cyclopentafllphenanthrene}

To $10 \mathrm{mmol}$ cyclopenta[l]phenanthrene, dissolved in $20 \mathrm{ml}$ of THF and cooled with an icebath, $10 \mathrm{mmol}$ of $\mathrm{n}-\mathrm{BuLi}$ as a $1.6 \mathrm{M}$ solution in hexane were added. The reaction mixture was stined at room temperature overnight and the solvents removed under reduced pressure. After rinsing two times with $20 \mathrm{ml}$ of pentane, the yellow to green residual solid was dissolved in $20 \mathrm{ml}$ of THF and treated at room temperature with $12 \mathrm{mmol}$ trimethylsilylchloride. The dark red solution was stirred again overnight, the solvent evaporated and the product extracted with $40 \mathrm{ml}$ of pentane from which it was obtained by evaporation of the solvent.

\section{3. i-Trimethylsily'-cyclopenta[l]phenanthrene}

Yield: 90\% (yellow oil). 'H-NMR $\left(\mathrm{CDCl}_{3} ; 250\right.$ MHz): $\delta 8.75-\{.70(\mathrm{~m}, 2 \mathrm{H}), 8.26-8.22(\mathrm{~m}, 1 \mathrm{H})$,
8.01-7.97 (m, 1 H), 7.65-7.53 (m, $4 \mathrm{H}+1 \mathrm{Cp}-\mathrm{H})$, 6.89-6.86 (m, 1 CpH), 4.35 (bs, I Cp-H), -0.05 (s, 9 TMS-H), MS (EI): $m / z 288\left(\mathrm{M}^{+}, 30 \%\right), 215\left(\mathrm{M}^{+}\right.$. TMS, 10\%), 73 (TMS, 100\%).

\subsection{I-Trimethylsilyl-2-methyl-cyclopentall]phenanth- rene}

Yield: $86 \%$ (yellow oil). 'H-NMR ( $\mathrm{CDCl}_{3} ; 250$ MHz): $\delta$ 8.71-8.64 (m, $2 \mathrm{H}), 8.15-8.12(\mathrm{~m}, 1 \mathrm{H})$, 7.88-7.85 (m, $1 \mathrm{H}), 7.63-7.48(\mathrm{~m}, 4 \mathrm{H}), 7.13(\mathrm{~s}, 1$ $\mathrm{CpH}$ ), 4.15 (s, I Cp-H), 2.35 (s, $3 \mathrm{Me}-\mathrm{H}),-0.08$ (s, 9 TMS-H), MS (EI): $m / z 302\left(\mathrm{M}^{+}, 40 \%\right), 228\left(\mathrm{M}^{+}\right.$. TMS, 10\%), 73 (TMS, 100\%).

\subsection{1-Trimethylsily/-2-phenyl-cyclopentallphenanth- rene}

Yield: $76 \%$ (beige solid). ${ }^{1} \mathrm{H}-\mathrm{NMR}\left(\mathrm{CDCl}_{3} ; 250\right.$ $\mathrm{MHz}): \quad \delta 8.73-8.68(\mathrm{~m}, 2 \mathrm{H}), 8.27-8.24(\mathrm{~m}, 1 \mathrm{H})$, 8.04-8.00 (m, $1 \mathrm{H}), 7.68-7.53$ (m, $4 \mathrm{H}+2 \mathrm{Ph}-\mathrm{H}), 7.70$ (s, 1 CpH), 7.45-7.39 (m, 2 Ph-H), 7.32-7.25 (m, 1 $\mathrm{Ph}-\mathrm{H}), 4.91$ (s, 1 Cp-H), -0.30 (s, 9 TMS-H), MS (EI): $m / z 364\left(\mathrm{M}^{+}, 20 \%\right), 291\left(\mathrm{M}^{+}-\mathrm{TMS}, 7 \%\right), 73$ (TMS, $65 \%)$.

3.6. General method for the synthesis of cyclopentall]phenanthrene titanium trichloride

1-Trimethylsilyl-cyclopenta[1]phenanthrene (amounts as obtained in the preceding reactions) was dissolved in $30 \mathrm{ml}$ of $\mathrm{CH}_{2} \mathrm{Cl}_{2}$ and treated with the equimolar amount of titanium tetrachloride at $0^{\circ} \mathrm{C}$. After stirring for $4 \mathrm{~h}$ at room temperature, the reaction mixture was concentrated to $15 \mathrm{ml}$ in vacuo and cooled to $-30^{\circ} \mathrm{C}$ overnight. Decantation of the mother liquor yielded the product as a red to purple solid.

\subsection{Cyclopentalllphenanthrene titanium trichloride (3)}

Yield: $53 \%$ red solid. ${ }^{1} \mathrm{H}-\mathrm{NMR}\left(\mathrm{CDCl}_{3} ; 600 \mathrm{MHz}\right)$ : $\delta 8.50 ́\left(\mathrm{~d}, J=8.0 \mathrm{~Hz}, \mathrm{C}_{4.5}-\mathrm{H}\right), 7.73-7.67\left(\mathrm{~m}, \mathrm{C}_{2.3 .6 .7^{-}}\right.$ H), $8.23\left(\mathrm{~d}, J=7.4 \mathrm{~Hz}, \mathrm{C}_{1.8}-\mathrm{H}\right), 7.62(\mathrm{~d}, J=3.3 \mathrm{~Hz}$, $\left.\mathrm{C}_{9.11}-\mathrm{H}\right), 7.25\left(\mathrm{t}, J=3.3 \mathrm{~Hz}, \mathrm{C}_{10}-\mathrm{H}\right)$, assignments of proton signals by ${ }^{13} \mathrm{C}$ and HMQC measurements, MS (EI): $m / z 370\left(\mathrm{M}^{+}, 7 \%\right), 333\left(\mathrm{M}^{+}-\mathrm{Cl}, 1 \%\right), 215(7-\mathrm{H}$, $100 \%$ ), Anal. Calcd for $\mathrm{C}_{17} \mathrm{H}_{11} \mathrm{TiCl}_{3}: \mathrm{C}, 55.26 ; \mathrm{H}, 3.00$. Found: C, 54.77; H, 3.22.

\subsection{2-Methyl-cyclopenta-[I]-phenanthrene titanium trichloride (4)}

Yield: $53 \%$ red needles. 'H-NMR $\left(\mathrm{CDCl}_{3} ; 600\right.$ $\mathrm{MHz}): \delta 8.54\left(\mathrm{~d}, J=7.9 \mathrm{~Hz}, \mathrm{C}_{4.5}-\mathrm{H}\right), 7.71-7.65(\mathrm{~m}$, $\left.\mathrm{C}_{2,3.6,7^{-}} \mathrm{H}\right), 8.18\left(\mathrm{~d}, J=7.6 \mathrm{~Hz}, \mathrm{C}_{1.8^{-}} \mathrm{H}\right), 7.47\left(\mathrm{~s}, \mathrm{C}_{9.11^{-}}\right.$ $\mathrm{H}), 2.73(\mathrm{~s}, \mathrm{Me}-\mathrm{H})$, assignments of proton signals by 
Table 3

Structure determination data for complex $5\left(\mathrm{C}_{17} \mathrm{H}_{11} \mathrm{Cl}_{3} \mathrm{Ti}\right)$

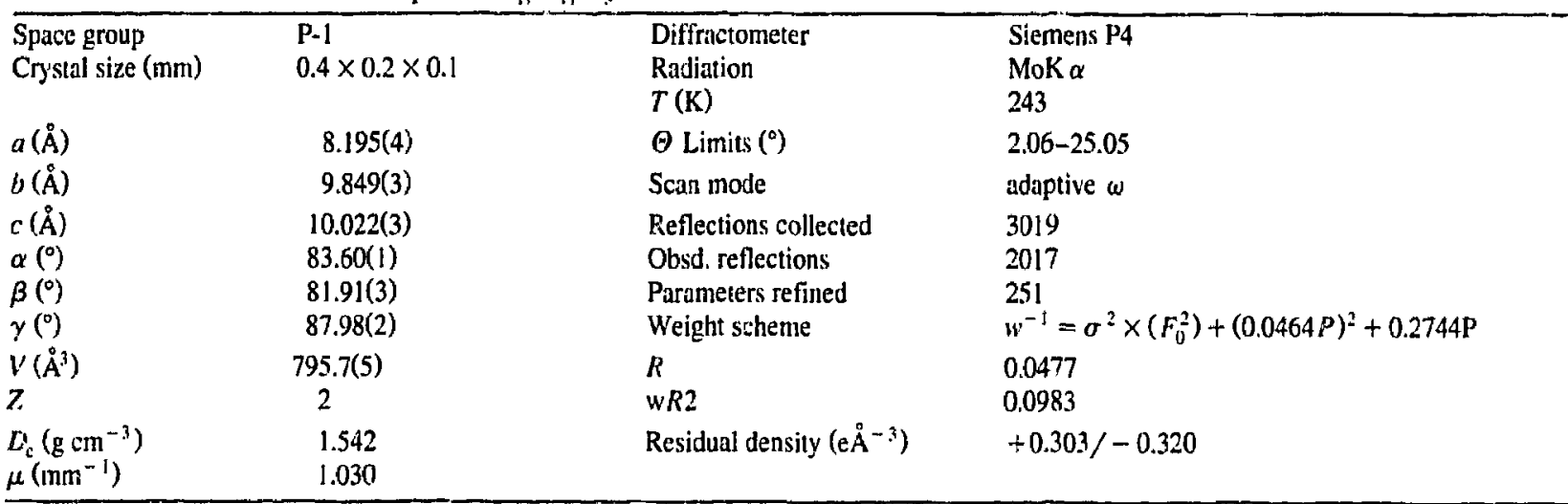

During data collection three check reflections every 100 reflections were measured. The structure was solved by direct methods asing the SHELX-86 program; for refinement the SHELX-93 program was used. All non-hydrogen atoms were refined anisotropic. Hydrogen atoms were taken from difference Fourier map and refined with fixed isotropic $U$.

${ }^{13} \mathrm{C}$, and HMQC measurements, MS (El): $m / z 384$ $\left(\mathrm{M}^{+}, 8 \%\right), 347\left(\mathrm{M}^{+} \cdot \mathrm{Cl}, 2 \%\right), 311\left(\mathrm{M}^{+}-2 \mathrm{Cl}, 2 \%\right), 229$ (8-H, 100\%), Anal. Calcd for $\mathrm{C}_{18} \mathrm{H}_{13} \mathrm{TiCl}_{3}: \mathrm{C}, 56.37$; $\mathrm{H}, 3.42$. Found: $\mathrm{C}, 55.98 ; \mathrm{H}, 3.54, \mathrm{X}$-ray structure data are given in Table 3; more information can be obtained at Fachinformationszentrum KarIsruhe, D-76344 Eggenstein-Leopoldshafen upon quotation of the depository number csb-406840, the authors and the journal reference of this article.

\subsection{2-Phenyl-cyclopentall]phenanthrene titanium trichloride (6)}

Yield: $66 \%$ red solid. ${ }^{1} \mathrm{H}-\mathrm{NMR}\left(\mathrm{CDCl}_{3} ; 600 \mathrm{MHz}\right)$ : $\delta 8.57\left(\mathrm{~d}, J=7.7 \mathrm{~Hz}, \mathrm{C}_{4.5^{-}} \mathrm{H}\right), 7.74-7.69\left(\mathrm{~m}, \mathrm{C}_{2.3 .6 .7^{-}}\right.$ H), $8.29\left(\mathrm{~d}, J=7.4 \mathrm{~Hz}, \mathrm{C}_{1.8}-\mathrm{H}\right), 8.03\left(\mathrm{~s}, \mathrm{C}_{9,11}-\mathrm{H}\right), 7.99$ (d, $J=7.4 \mathrm{~Hz}, 2 \quad o-\mathrm{Ph}-\mathrm{H}), 7.54(\mathrm{t}, J=7.4 \mathrm{~Hz}, 2$ $m-\mathrm{Ph}-\mathrm{H}), 7.46(\mathrm{t}, J=7.4 \mathrm{~Hz}, p-\mathrm{Ph}-\mathrm{H})$, assignments of proton signals by ${ }^{13} \mathrm{C}$, COSY and $\mathrm{HMQC}$ measurements, MS (El): $m / z 446\left(\mathrm{M}^{+}, 6 \%\right), 291$ (9-H, 100\%), Anal. Calcd for $\mathrm{C}_{23} \mathrm{H}_{15} \mathrm{TiCl}_{3}$ : C, 61.99; $\mathrm{H}, 3.39$. Found: C, $61.67 ; \mathrm{H}, 3.80$.

\subsection{Polymerization procedure for syndiotactic polystyrene}

A 300-ml flask was charged with $50 \mathrm{ml}$ of toluene, 5 $\mathrm{ml}(44 \mathrm{mmol})$ of freshly distilled styrene and $6 \mathrm{ml}$ of MAO under an argon atmosphere. The flask was placed in an oil bath at the desired temperature and stirred for $10 \mathrm{~min}$. The titanium catalyst $(2.5 \mu \mathrm{mol}, 1 \mathrm{ml}$ of a 2.5 $\mathrm{mM}$ solution in toluene) was added via syringe and the reaction was stirred for 10 to $20 \mathrm{~min}$. The reaction mixture was hydrolyzed by addition of $10 \% \mathrm{HCl}$ in methanol, filtered, washed with excess methanol and dried over night at $100^{\circ} \mathrm{C}$. The polymer was extracted with 2-butanone for $24 \mathrm{~h}$ in a Soxhlet extractor to remove atactic polymer and dried again at $100^{\circ} \mathrm{C}$ for 24 h.

\section{Acknowledgements}

The authors thank Monika Cavegn for valuable help with NMR-experiments, BASF AG Ludwigshafen for DSC and GPC measurements and financial support, and the Landesgraduiertenförderung Baden-Wiirttemberg for a fellowship.

\section{References}

[1] N. Ishihara, T. Seimiya, M. Kuramoto, M. Uoi, Macromolecules 19 (1986) 2464; N. Ishihara, M. Kuramoto, M. Uoi, Macromolecules 21 (1988) 3356.

[2] D.J. Gillis, M.-J. Tudoret, M.C. Baird, J. Am. Chem. Soc. 115 (1993) 2543; R. Quyoum, Q. Wang, M.-J. Tudoret, M.C. Baird, J. Am. Chem. Soc. 116 (1994) 6435; Q. Wang, R. Quyoum, D.J. Gillis, M.-J. Tudoret, D. Jeremic, B.K. Hunter, M.C. Baird, Organometallics 15 (1996) 693; C. Pellecchia, A. Proto, P. Longo, A. Zambelli, Makromol. Chem. Rapid Commun. 13 (1992) 277; C. Pellecchia, P. Longo, A. Proto, A. Zambelli, Makromol. Chem. Rapid Commun. 13 (1992) 265; C. Pellechia. D. Pappalardo, L, Oliva, A. Zambelli, J. Am. Chem. Soc. 117 (1995) 6593.

[3] A. Zambelli, C. Pellecchia, L. Oliva, P. Longo, A. Grassi, Makromol. Chem. 192 (1991) 223; C. Pellecchia, P. Longo, A. Grassi, P. Ammendola, A. Zambelli, Makromol. Chem. Rapid Commun. 8 (1987) 277; J.C.W. Chien, J. Salajka, S. Dong, Macromolecules 25 (1992) 3199; P. Longo, A. Grassi, A. Proto, P. Ammendola, Macromolecules 21 (1988) 24: A. Zambelli, P. Longo, C. Pellecchia, A. Grassi, Macromolecules 20 (1987) 2035; A. Grassi. P. Longo, A. Proto, A. Zambelli, Macromolecules 22 (1989) 104;

[4] H.H. Brintzinger, D. Fischer, R. Mülhaupt, B. Rieger, R. Waymouth, Angew. Chem. 107 (1995) 1255; W. Kaminsky, Macromol. Chem. Phys. 197 (1996) 3907.

[5] T.E. Ready, R.O. Day, J.C.W. Chien, M.D. Rausch, Macromolecules 26 (1993) 5822. 
[6] P. Foster, J.C.W. Chien, M.D. Rausch, Organometallics 15 (1996) 2404.

[7] N. Schneider, M.E. Huttenloch, U. Stehling, R. Kirsten, F. Schaper, H.H. Brintzinger, Organometallics, in press.

[8] D.J. Duncalf, H.J. Wade, C. Waterson, P.J. Derrick. D.M. Haddleton, A. McCamley, Macromolecules 29 (1996) 6399.
[9] J.M. Hawkins, S. Loren, J. Am. Chem. Soc. 113 (1991) 7794. [I0] A.C. Cope, L. Field, D.W.H. MacDowell, M.E. Wright, J. Am. Chem. Soc. (1956) 2547; B. Eliasson, M.H. Nouri-Sorkhabi, L. Trogen, I. Sethoson, U. Edlund, A. Sygula, M. Rabinowitz, J. Org. Chem. 54 (1989) 171. 